\title{
Strates
}

STRATES Matériaux pour la recherche en sciences sociales

Hors-série | 2002

Parcours dans la recherche urbaine, Michel Rochefort, un géographe engagé

\section{La place de Michel Rochefort dans la recherche urbaine française}

Jacques Brun et Catherine Paix

\section{(2) OpenEdition}

Journals

Édition électronique

URL : http://journals.openedition.org/strates/505

DOI : $10.4000 /$ strates.505

ISSN : $1777-5442$

Éditeur

Laboratoire Ladyss

Édition imprimée

Date de publication : 1 janvier 2002

ISSN : 0768-8067

Référence électronique

Jacques Brun et Catherine Paix, "La place de Michel Rochefort dans la recherche urbaine française », Strates [En ligne], Hors-série | 2002, mis en ligne le 22 avril 2005, consulté le 08 septembre 2020. URL : http://journals.openedition.org/strates/505 ; DOI : https://doi.org/10.4000/strates.505

Ce document a été généré automatiquement le 8 septembre 2020

Tous droits réservés 


\title{
La place de Michel Rochefort dans la recherche urbaine française
}

\author{
Jacques Brun et Catherine Paix
}

1 Le paradoxe de Michel Rochefort est d'être célèbre et relativement méconnu à la fois. Célèbre par ses travaux sur les réseaux urbains et parce qu'il s'est imposé comme le leader d'une géographie «volontaire » soucieuse de donner une application pratique à la recherche. Relativement méconnu, parce qu'il fait partie de ces universitaires qui ont fait passer une grande partie de leur réflexion théorique dans leurs enseignements et leurs directions de recherche, que ses convictions profondes et ses orientations marxistes l'ont souvent mis en porte-à-faux par rapport à l'establishment, et que son œuvre écrite, à la fois ramassée et synthétique, ne permet pas toujours aisément de discerner les principales avancées auxquelles il a personnellement contribué. Alors qu'il a ouvert la voie à des recherches parmi les plus marquantes des cinquante dernières années dans le domaine urbain, les fondements théoriques de sa démarche et la complexité de sa pensée ont été, de ce fait, en partie occultés. Et lui-même ne les a guère explicités, quand il n'a pas contribué, à travers ses propres remises en cause, à masquer la richesse de son travail. Il est ainsi remarquable de constater que son nom et ses travaux ne soient aujourd'hui que rarement mentionnés, y compris dans des ouvrages qui font retour sur l'évolution de la géographie et de la recherche urbaine ${ }^{1}$. Sans doute parce que son rayonnement et son influence dans la discipline ont été trop fortes. Mais aussi parce que Michel Rochefort est un homme de science, profondément animé par le doute, qui s'est toujours défié des images lisses du prêt-à-porter théorique, et qui a toujours préféré le questionnement aux résultats éphémères et aux effets de mode, la méthode et l'administration de la preuve aux généralisations hâtives et aux dogmes.

2 S'il retrace dans ce livre les grandes étapes de son parcours, et si l'on peut mieux percevoir dans ses derniers ouvrages les lignes majeures de sa réflexion, il importe donc de rappeler les points forts de sa démarche intellectuelle et de ses travaux en les resituant dans l'évolution de la géographie et de la recherche urbaine. On tentera ainsi de leur donner plus de visibilité, et d'en montrer les progressions et la portée, ce dont 
son témoignage, empreint de modestie et très lapidaire, ne rend qu'imparfaitement compte.

3 Trois constantes sont à souligner, car elles ont beaucoup contribué à faire avancer la géographie et participent aux grands débats théoriques et méthodologiques qui ont animé les sciences sociales au cours de ce dernier demi-siècle. C'est d'abord le souci de relier les phénomènes observés aux évolutions globales de la société, et l'ambition de construire un système de référence permettant d'appréhender les mécanismes d'organisation et de différenciation de l'espace, dans leurs régularités, mais aussi dans leur diversité et leur complexité, en donnant des bases rigoureuses au travail de comparaison. En écho à des approches largement portées par l'idée de totalité chère aux marxistes, Michel Rochefort a par ailleurs beaucoup contribué au développement de la recherche pluridisciplinaire. Que ce soit dans ses propres travaux ou à travers les nombreux programmes qu'il a impulsés, il n'a cessé de pratiquer et d'encourager les confrontations avec les économistes, les sociologues, les démographes, les urbanistes, etc. - en un mot d'ouvrir les frontières entre les disciplines - et de développer la recherche collective, en particulier au sein de son laboratoire. Enfin, et ceci n'est dissociable, ni de sa sensibilité aux problèmes humains, ni de ses engagements idéologiques, c'est sur des problèmes parmi les plus aigus des sociétés contemporaines et sur les inégalités socio-spatiales, qu'il a porté son attention, avec pour préoccupation de donner des outils intellectuels permettant, sinon de les corriger, du moins de les comprendre et de les dénoncer. Et s'il compte parmi ceux qui ont cherché à mettre leur travail au service de l'action, et même goûté à la tentation technocratique, il a toujours marqué sa volonté de ne pas subordonner la recherche fondamentale aux commandes du pouvoir.

Les débuts d'un parcours dans leur contexte

4 Michel Rochefort a relaté son engagement dans la géographie et la recherche urbaine, et plus précisément dans l'étude des rapports entre armatures urbaines et organisation régionale. Le contexte dans lequel il a effectué ce choix thématique appelle cependant quelques précisions qui aideront peut-être à mieux comprendre l'orientation qu'il a donnée à sa thèse.

Ses recherches ont été d'emblée animées, on le sait, par l'idée de rendre compte des processus et mécanismes socio-économiques de différenciation et d'organisation de l'espace, et elles s'inscrivent très clairement dans le courant des travaux d'inspiration marxiste ${ }^{2}$ d'après-guerre : ceux notamment de ses maîtres, André Cholley, Jean Dresch, et surtout Jean Tricart et Pierre George, et ceux des historiens des Annales ${ }^{3}$ qui placent au centre de leur analyse les évolutions structurelles de l'économie et de la société et les rapports sociaux. Mais il est vrai que sa thèse, L'organisation urbaine de l'Alsace, n'est pas totalement exempte d'une certaine contradiction entre son marxisme et une approche qu'il a lui-même qualifiée de fonctionnaliste et dont il a montré les limites par la suite. Dans son mémoire de DES sur la campagne autunoise et ses bordures morvandelles ${ }^{4}$, il s'était attaché à expliquer les différenciations agraires à partir d'une analyse historique de l'évolution des structures et des rapports sociaux et économiques, dont il avait reconstitué les principales étapes en les reliant à la pénétration différenciée des capitaux bourgeois et aux fluctuations et transformations plus globales de l'économie. À travers ce travail, dans lequel il mettait en évidence les conditions et modalités d'expansion du capitalisme dans l'espace étudié et les rapports villes/ campagnes qui en sont constitutifs, il avait donc abordé de front la question des 
rapports entre les formes de développement du système économique et social et les structures de l'espace, voire plus précisément celle de l'articulation des modes de production et de la différenciation des formations socio-spatiales qui leur sont associées. Celle aussi des rapports contradictoires et de la diversité des temporalités qui assurent à chaque système socio-spatial sa singularité et sa dynamique propre.

Dans sa thèse, il reprend la même démarche historique, en l'appliquant cette fois à l'organisation urbaine régionale. Il consacre ainsi une grande partie de son travail à mettre en évidence les mécanismes socio-économiques de « mise en place » des réseaux urbains actuels aux différents temps d'évolution du système capitaliste dans la région étudiée. Mais son questionnement de départ, « analyser les modalités du rôle des villes dans l'espace régional et les facteurs d'explication de cette organisation actuelle, [...] chercher dans une région déterminée comment et sous la pression de quels facteurs les villes se localisent, s'organisent entre elles, se hiérarchisent et se diversifient pour répondre aux besoins de cette région ", le conduit à accorder beaucoup d'importance à l'étude de l'organisation urbaine elle-même. Ses efforts se portent d'abord sur la recherche des variables économiques qui définissent les villes et leur position respective, et sur l'analyse des relations qui unissent les villes entre elles et à leur environnement extérieur et qui structurent l'espace régional. S'il met en évidence les principes qui régissent la hiérarchisation des villes et leurs zones d'influence, et s'il élabore une méthode de classification fonctionnelle des villes, il met donc plus l'accent sur la fonctionnalité des villes dans l'économie régionale, - en d'autres termes, sur la structure des activités et le fonctionnement de l'économie dans l'espace -, que sur le système économique et la dynamique des rapports sociaux ${ }^{5}$. Et par l'attention qu'il porte aux configurations et au fonctionnement du système urbain, son analyse n'est pas non plus dénuée d'un certain «spatialisme ».

7 On a eu cependant trop vite fait de ne retenir que cet aspect de sa recherche et d'en faire la critique après coup, sans réfléchir suffisamment aux raisons qui l'ont orienté dans cette voie et pour lesquelles il était malaisé à l'époque de ne pas succomber à cette " dérive ${ }^{6}$.

8 Les questions posées à la géographie dans les années 50 , et le conformisme universitaire et disciplinaire de l'époque, y sont sans doute pour beaucoup. Il serait en effet anachronique de penser qu'au début des années 50 un jeune chercheur bénéficiait d'une entière liberté dans le choix de son sujet de thèse et qu'il disposait de la même latitude qu'aujourd'hui pour orienter ses recherches. Michel Rochefort, qui s'est toujours gardé d'enfermer ses élèves dans un cadre hypothétiquement spécifique à la géographie, fait justement partie de ceux de sa génération qui ont le plus oeuvré dans le sens de cette ouverture.

9 Pour pouvoir concrètement se déclarer "géographe ", occuper un poste d'assistant, déposer et soutenir une thèse de géographie, il fallait, en premier lieu, adopter un thème et une démarche justifiant la qualification de "géographiques". Le souci de l'identité de la géographie, récurrent dans la discipline, restreignait la liberté des chercheurs, et cela d'autant plus peut-être que celle-ci était en réalité mal définie, et qu'en dépit des nombreux débats et textes visant à la cerner, elle ne faisait pas l'objet d'un véritable consensus. L'autonomie de la géographie par rapport à l'histoire était par ailleurs très récente et incomplète ${ }^{7}$, et le tiraillement entre la géographie physique, alors quasi-totalement dominée par la géomorphologie structurale, et la géographie humaine, était le plus souvent résolu par des affirmations volontaristes sur l'unité de la 
discipline. Mais dans le sillage des historiens des Annales ${ }^{8}$, et de Lucien Febvre en particulier, les confrontations disciplinaires avaient parallèlement donné lieu, depuis l'avant-guerre, à un effort important de renouvellement théorique et méthodologique de la géographie humaine, et à un travail réflexif visant à mieux en délimiter l'objet et les méthodes 9 .

Il existait donc une certaine hiérarchie dans la légitimité des thèmes de recherche et des démarches pouvant donner accès à la reconnaissance académique. Celle-ci n'aurait sans doute pas été accordée aisément à une thèse trop expressément centrée sur les rapports et a fortiori les antagonismes entre les groupes sociaux. Michel Rochefort dira plus tard que du fait « qu'il fallait jouer le jeu de la thèse, [...] il avait pris un sujet qui se prêtait peu à la confrontation avec les problèmes sociaux, et qu'il avait hésité dans l'interprétation des faits [...], qu'il ne les avait pas référés à un système plus général, et qu'il était resté très inductif $[. ..] \aleph^{10}$. Certes, il serait faux de penser que les géographes n'accordaient pas d'importance aux structures et formes d'organisation sociales et aux changements d'ensemble de la société. Nombre d'entre eux les considéraient comme partie intégrante de leurs analyses et cherchaient à rendre compte des processus socioéconomiques d'élaboration et d'organisation de l'espace, en les rattachant à des évolutions plus globales. Le mémoire de Michel Rochefort s'inscrit dans cette lignée. Mais, dans leur ensemble, les géographes accordaient encore une grande place à l'étude des paysages et de leur genèse et aux rapports homme/milieu, et même lorqu'ils affirmaient le rôle déterminant de l'économie, leurs recherches s'attachaient surtout, sur la base d'une analyse descriptive, aux configurations spatiales elles-mêmes et à l'inscription des structures économiques et sociales dans l'espace ${ }^{11}$. Dans la filiation vidalienne, les échelles locale et régionale étaient également privilégiées. À cela s'ajoutait le fait que, pour une partie de l'establishment, toute approche un peu trop fouillée des structures sociales était suspectée de ne pas être du ressort de la géographie et ceci d'autant que des géographes de renom étaient engagés au parti communiste ${ }^{12}$. Quant à la dimension politique des questions abordées, elle était très souvent considérée comme étrangère au champ de la géographie. Plusieurs des maîtres de Michel Rochefort, André Cholley, Jean Dresch, mais aussi Pierre George qui a dirigé sa thèse, sont ainsi marxistes et fermes défenseurs d'une vision de la géographie qui impose un certain nombre de carcans, dont le primat de la description empirique par rapport à l'analyse des processus historiques, et celui des phénomènes de "régionalisation » et d'organisation spatiale par rapport aux tensions et conflits entre les groupes.

11 Inversement, mettre l'accent sur les relations villes/campagnes, et plus explicitement sur le rôle des villes dans la structuration de l'espace régional, n'était pas mal venu, même si les paysages ne tenaient qu'une place succincte dans l'analyse. L'étude des rapports villes/campagnes était en effet un des thèmes importants de la géographie française de l'époque. De nombreux travaux, tel le mémoire de Michel Rochefort sur le rôle de la bourgeoisie urbaine dans l'évolution des structures agraires et des systèmes de culture de la plaine d'Autun, mettent alors en évidence le rôle des villes et des capitaux urbains dans la transformation du monde rural et de l'agriculture. Il existait par ailleurs au sein de la géographie tout un vieux courant de pensée qui rattachait l'étude des villes à celle de la circulation et des échanges. Plus rares étaient les travaux sur les liens entre ville et industrie. La recherche urbaine était tantôt rangée dans la géographie de l'habitat, tantôt dans celle de « la vie de relations », et tous les classiques insistaient sur les relations entre la ville et le monde extérieur, notamment la région 
dont elle fait partie, et dont elle est la capitale ou le centre. Or c'est très précisément un des thèmes à partir duquel s'engage après-guerre un effort de réflexion plus systématique. À la suite des travaux de Raoul Blanchard sur Grenoble, qui avait mis l'accent sur le rôle de "commandement» de la ville et sur l'aire d'influence de ses activités commerciales et de services ${ }^{13}$, et surtout de Georges Chabot, qui avait avancé l'idée de hiérarchie et d'organisation urbaine structurant l'espace régional et affirmé plus clairement le rôle moteur des villes dans l'organisation de l'espace ${ }^{14}$, l'analyse des relations entre les grands centres urbains et leurs périphéries et celle des zones d'influence et d'attraction urbaines avaient été amorcées. Puis dans l'immédiat aprèsguerre, moment où se renforce la prise de conscience des changements liés à la croissance urbaine, ces recherches avaient fait l'objet d'essais de formalisation théorique et les géographes avaient abordé plus frontalement la question du développement urbain et de ses rapports avec l'économie. Georges Chabot consacre alors un chapitre de son précis Les villes au "rôle des villes dans le Pays", où il développe la notion de réseau urbain ${ }^{15}$; Jean Gottmann reprend la question des systèmes de relations qui, à partir des villes, animent l'économie régionale et fait le lien entre les besoins de la région, la consommation et le développement urbain ${ }^{16}$. De son côté, Jean Dresch consacre plusieurs articles à la croissance des villes africaines qu'il relie au système d'exploitation coloniale ${ }^{17}$, tandis que Pierre George, dans son ouvrage de 1952, La ville, le fait urbain à travers le monde, s'attache plus largement à dresser un tableau des formes d'urbanisation selon les caractéristiques des systèmes économiques et sociaux.

Dans le domaine urbain, la géographie opérait donc une montée en généralité, et une "nouvelle géographie " commençait à émerger, toujours très attentive aux formes urbaines, mais dégagée de l'exceptionnalisme géographique, moins axée sur l'étude des relations homme/milieu, et davantage orientée sur celle des rapports entre système économique et système d'organisation spatiale. Il faut bien voir cependant que, mis à part quelques articles et manuels, cette orientation n'en était qu'à ses débuts et que la recherche urbaine n'occupait encore qu'une place très limitée dans la discipline. La géographie s'était en effet principalement adonnée jusque-là à de grandes synthèses régionales, et à des monographies historiques de villes, et plus particulièrement de grandes villes, et elle consacrait plus de pages aux études rurales qu'urbaines ${ }^{18}$. Par ailleurs, si les relations villes/région avaient été explorées, les recherches sur la question demeuraient très fragmentaires et dispersées, et leurs fondements théoriques et méthodologiques peu assurés. Quant à la notion de réseau urbain comme principe d'organisation régionale, elle n'avait encore donné lieu à aucune analyse approfondie. Elle impliquait l'idée qu'à partir de l'étude des villes et de leurs relations il est possible de rendre compte des modalités d'inscription du système économique dans l'espace et d'appréhender la dynamique des systèmes régionaux. Il n'est donc pas très étonnant qu'un géographe marxiste, intéressé par l'essor des villes et curieux de comprendre les disparités spatiales du développement économique et social, n'ait pas axé d'emblée ses investigations sur les relations ville/industrie, même si Pierre George s'efforçait de faire des « rapports de production » le paradigme d'une nouvelle géographie.

Dans sa thèse, Michel Rochefort n'en néglige d'ailleurs pas du tout l'analyse. On peut ajouter que, plus que l'étude de la dynamique intra-urbaine ou que l'analyse sectorielle de l'économie, la question du rôle des villes dans l'organisation régionale, qui permet une approche globale des rapports société/espace, correspond à ses préoccupations. Elle touche également très directement à l'un des grands problèmes politiques de 
l'époque: l'aménagement régional. La volonté de modernisation de l'économie française et celle de rééquilibrer le territoire, qui sont alors indissociables, interpellent en effet très directement les géographes sur cette question, et cela d'autant que l'idée de planification fait l'objet d'un consensus entre les grands courants politiques. C'est d'ailleurs ce qui a sans doute incité Pierre George, - qui était soucieux d'une géographie plus directement en prise avec les problèmes de société, et avait déjà participé à des travaux destinés à l'aménagement du territoire ${ }^{19}-$, à engager plusieurs de ses élèves dans de grandes thèses sur le sujet. Et l'un des objectifs annoncés d'entrée de jeu par Michel Rochefort dans son travail est de donner « des clés » pour l'aménagement, ce qui, par bien des aspects, en a fait le succès.

La première grande thèse française sur le rôle des villes dans l'organisation régionale

Sa thèse, L'organisation urbaine de l'Alsace ${ }^{20}$, a eu, on le sait, un retentissement considérable ${ }^{21}$. Son étude des réseaux urbains représentait en effet un effort important de formalisation méthodologique et de systématisation du rôle des villes à l'échelle d'une grande région, qui était nouveau en géographie et répondait aux préoccupations d'aménagement du territoire de l'époque. Elle a cependant donné lieu par la suite à quelques malentendus, et, du fait même de son succès, on en a trop souvent gardé une image déformée. À quarante ans de distance, il n'est donc pas inutile d'en refaire le bilan, en replaçant cette recherche dans les questionnements des années 50 et 60 , et de souligner ce qu'elle ouvrait comme nouvelles perspectives et ce qu'elle préfigurait de ses orientations ultérieures.

La notoriété de la thèse dans les années qui suivent sa publication tient d'abord au fait qu'il s'agit de la première des grandes thèses françaises de géographie explicitement centrées sur le rôle des villes dans l'organisation régionale. Celle de Jean Labasse, Les capitaux et la région, publiée quelques années auparavant, a certes joué un rôle important dans l'évolution de la pensée des géographes et autres spécialistes d'aménagement, et le rayonnement en a été renforcé par les responsabilités que son auteur a exercées dans le domaine de la politique régionale. Mais elle était strictement consacrée à l'évolution des réseaux bancaires régionaux, et on peut se demander si sa démarche n'a pas été considérée comme n'étant pas "authentiquement» géographique, à une époque où le respect de l'identité de la discipline était, comme on l'a vu, particulièrement prégnant ${ }^{22}$. À l'inverse, celle de Michel Rochefort offrait une grille d'analyse globale des relations entre les villes et leurs aires d'influences, et en brossant d'entrée de jeu un tableau des configurations spatiales contemporaines du rapport villes/campagnes, en donnant une méthode de description empirique rigoureuse de ces relations, et en abordant les principaux aspects de la vie économique régionale, elle était plus conforme aux objectifs classiques de l'analyse géographique. Elle pouvait également faire office de «modèle » dans le domaine, alors naissant, de la géographie appliquée. De là son audience rapide au ministère de l'Équipement. Si la thèse de Raymond Dugrand, soutenue quelque temps plus tard, contient également une analyse très fouillée des aires d'influence des villes et de la structure d'un réseau régional, c'est en effet à L'organisation urbaine de l'Alsace que l'on se réfère dans la première partie des années 60 , pour jeter les bases d'une nouvelle organisation territoriale dont les villes doivent constituer « l'armature ». Comme celui d'organisation, à l'époque souvent confondu avec l'aménagement volontaire, ce terme d'armature qu'il utilise pour illustrer la structure du système urbain, a contribué à conforter sa notoriété. Métaphore, autant que concept analytique, l'expression se prête en effet parfaitement à un emploi dans des travaux de prospective sur l'aménagement du 
territoire, car elle renvoie à un modèle généralisable, en d'autres termes à une image simplifiée de l'organisation urbaine: "Le développement progressif de la concentration économique a façonné une armature urbaine hiérarchisée dont l'unité n'est plus la ville, mais le réseau régional. Celui-ci repose sur la combinaison de trois éléments : le centre régional, les centres de sous-région, les centres locaux; cette structure détermine les trois catégories fondamentales d'une classification urbaine [...]. Tout programme d'aménagement [...] urbain [...] doit s'appuyer sur une juste appréciation de la place de la ville dans le réseau urbain, place qui commande les modalités mêmes de cet aménagement. [...] la recherche d'une organisation régionale rationnelle doit dominer les particularités individuelles de chaque agglomération et [...] s'efforcer de corriger les malformations et inadéquations intrinsèques du réseau actuel ou viser à transformer ce réseau en vue de l'adapter à de nouvelles perspectives de l'économie régionale ${ }^{23}$. Pour les fonctionnaires engagés à cette époque dans un travail d'élaboration d'outils de planification territoriale, Michel Rochefort apparait donc comme le spécialiste qui leur fournit à la fois les outils méthodologiques et conceptuels, mais aussi la garantie scientifique dont ils ont besoin.

Les déphasages entre le contenu effectif des trois thèses évoquées ici, et l'image qui en a été retenue par la majorité des géographes appellent d'ailleurs réflexion. Dans les années 60 , que l'on soit de droite ou de gauche, on adhère à l'idée que la "région » est une réalité majeure du développement, et on pense que les « capitales régionales » sont ou doivent être les pôles moteurs de la croissance régionale, quand bien même le dynamisme de ces villes ne va pas dans ce sens et la réalité économique est en train de devenir supra-régionale. Cette représentation n'est pas étrangère aux grandes orientations des instances d'aménagement du territoire, et aux travaux d'économie régionale menés à partir des années 50 . L'influence des économistes, et la diffusion du concept de région polarisée, y sont pour beaucoup ${ }^{24}$. Leurs théories confortent en effet l'idée que l'intégration des structures économiques régionales à partir d'un pôle moteur, et les économies d'échelles et effets d'entraînement qui lui sont liées, sont la condition de la croissance et du développement. Bien qu'elles évacuent souvent de l'analyse les dimensions socio-historiques qui assurent aux dynamiques régionales leur singularité, elles opèrent une séduction sur les esprits soucieux d'une meilleure répartition de la croissance. Elles donnent aussi du poids aux travaux des géographes, comme ceux de Michel Rochefort. Ceux-ci, en effet, mettent en évidence les principes de répartition et de hiérarchisation des activités dans l'espace ainsi que les délimitations régionales et rendent opératoires les théories de localisation et de centralité urbaine. Toute la politique des «métropoles d'équilibre » à laquelle Michel Rochefort participe à la suite de sa thèse, est fondée sur les notions de pôle régional et de région polarisée qui avalisent l'idée que « l'existence de pôles économiques intégrés est la condition même de l'intégration de l'économie nationale ${ }^{25}$.

On a ainsi longtemps vu dans la thèse de Jean Labasse un plaidoyer en faveur de l'idée que, pour être "dynamique», une région économique "moderne» devait être organisée selon une structure en réseau. Surtout, on a cru qu'il montrait que les capitaux régionaux jouaient un rôle essentiel dans cette dynamique, à travers le rayonnement d'une métropole bancaire capable de susciter l'innovation et l'investissement. Or son travail montre, au contraire, comment un réseau bancaire régional est intégré - sous peine de disparition - dans une structure d'échelle 
nationale, et comment les capitaux régionaux sont pompés par la métropole nationale et redistribués selon une logique qui échappe au contrôle de la bourgeoisie locale.

De la thèse de Raymond Dugrand on a retenu qu'une région dépourvue de vraie métropole, et dont le réseau urbain n'est pas hiérarchisé se laisse « coloniser » par les capitaux « étrangers » (ceux de Paris, Lyon etc.) et entre inévitablement en crise. On a retenu aussi que, selon sa propre conclusion, Marseille devrait assumer son rôle moteur de capitale de la région méditerranéenne. Toutes hypothèses infirmées ultérieurement par le développement de Montpellier, dont le dynamisme ne doit pas grand chose au capitalisme régional. Là encore, on a apprécié ce qui entrait en résonance avec la problématique des milieux technocratiques, fût-ce avec une volonté de contestation politique, plus que ce qui constitue l'apport décisif de l'ouvrage, son analyse historique. Quant à la thèse de Michel Rochefort, les contemporains, trop obnubilés par l'idéologie technocratique dominante, en ont surtout retenu la notion d'armature urbaine et une méthode qui permet de classer les pôles urbains et leurs aires d'influence et d'identifier à partir de là les centres d'impulsion de la vie économique régionale. Et il semble bien que cela ait contribué à faire oublier que sa thèse comporte, à côté de l'étude formelle de la structure spatiale du réseau, une analyse très poussée de l'évolution de l'économie régionale, qui le conduit dans sa conclusion à souligner que "si le réseau urbain régional [...] semble constituer l'indispensable armature technique de la vie régionale ", la concentration économique et financière de la première moitié du $\mathrm{XX}^{\mathrm{e}}$ siècle, " $\mathrm{a}$ progressivement démantelé son autonomie $»^{26}$. Quoi qu'il en soit, le travail méthodologique qui sous-tend sa recherche, apportait effectivement des réponses à des questions théoriques et pragmatiques parmi les plus importantes que se posaient les spécialistes à cette époque. Les éléments de cette adéquation entre le contenu de la thèse et une "demande sociale", que relaie la réflexion d'un certain nombre d'économistes et de géographes, se retrouvent en de nombreux points de l'analyse.

La thèse aborde, quasi d'entrée de jeu, toute une série de questions concernant le découpage territorial. Certes, il existait, on l'a vu, tout un corpus de textes sur les rapports entre l'aire d'influence des villes et la délimitation des régions et sousrégions ${ }^{27}$. Mais Michel Rochefort est le premier à leur consacrer une analyse systématique, à l'échelle d'une grande région. Et de plus, son analyse n'est pas, à la différence d'autres études, antérieures ou ultérieures, une fin en soi. Sa fonction est d'introduire à une étude globale de la totalité des problèmes touchant au développement économique régional. L'étude des zones d'influence urbaine à laquelle il procède dans la première partie de sa thèse est avant tout un moyen pour identifier les fonctions économiques qui sont à la base de l'organisation urbaine, et leurs délimitations font l'objet d'une analyse très exhaustive concernant les activités de distribution des biens et des services, et le rôle des villes dans l'organisation de la production agricole et industrielle. C'est ce qui lui permet d'élaborer une classification fonctionnelle de l'ensemble des agglomérations dans la région étudiée, et de décrire les réseaux urbains. C'est à partir de là également qu'il peut définir avec précision les limites régionales et subrégionales d'influence des principales villes, réseaux de villes, et sous-secteurs de l'organisation urbaine qui structurent la vie régionale. La thèse donne donc à la fois une méthode générale pour appréhender les différents niveaux de la hiérarchie urbaine et l'ensemble des rapports et liens de dépendance qui unissent les villes entre elles et assurent à la région sa cohésion, et une grille de lecture pour analyser la spécificité de leur rôle dans une région donnée. C'est sans doute pourquoi sa thèse va servir de référence à de nombreux travaux de statuts divers: mémoires 
universitaires, articles de revues géographiques, mais aussi études commandées par l'administration en charge de l'aménagement du territoire.

L'autre nouveauté essentielle, sur laquelle on n'a pas suffisamment insisté, réside dans l'effort de mesure, dans un domaine où celle-ci était assez peu pratiquée. Là encore, il est vrai qu'il n'y a pas lieu de parler de rupture: dans la géographie des relations, l'emploi des chiffres n'est pas tout à fait nouveau. Ce n'est pas, d'autre part, sur le plan de la nature des données que Michel Rochefort innove le plus. Sa thèse n'apporte que des informations partielles sur la consommation, l'investissement, ou encore sur les tensions entre les groupes sociaux. Mais le recours à une enquête systématique, dans la totalité des communes d'un ensemble régional, était encore exceptionnel en géographie, mise à part la géographie de la population, qui bénéficie de sources écrites et de recensements. La méthode qu'il emploie repose sur le recueil et l'élaboration de données statistiques en vue d'identifier avec précision des relations, des limites, et des hiérarchies, et elle tranche avec les études de géographie traditionnelle. Elle bouscule également, du fait qu'elle se démarque de tout inventaire, une tradition de la géographie, car elle est strictement centrée sur les données qui peuvent être utiles à la démonstration.

Quant aux critères retenus pour définir la structure du réseau et l'aire d'influence des villes - critères relevant du domaine des échanges, bien plus que de l'activité productive et surtout de l'investissement du capital -, il faut bien voir que l'importance qu'il a donnée à cette série d'indicateurs s'inscrit à l'époque dans un mouvement de modernité de la problématique géographique. Certes, l'analyse des structures spatiales liées aux échanges n'est pas en soi un thème radicalement nouveau. Les rapports entre les villes, depuis les plus grandes jusqu'aux simples bourgs, et l'espace extérieur, proche ou lointain, étaient déjà des objets privilégiés de la géographie urbaine. Et il a pu sembler paradoxal de la part d'un marxiste d'avoir autant privilégié les fonctions tertiaires et les configurations spatiales des échanges, plutôt que les structures productives. Mais en réalisant une recherche systématique sur la sphère des services, à une époque où la taxinomie de Colin Clark n'était pas encore vulgarisée en France, et où l'on commençait seulement à prendre conscience du rôle, non plus seulement passif, mais actif des activités tertiaires dans la croissance, la thèse de Michel Rochefort contribue à l'aggiornamento de la géographie économique. Si, d'une certaine façon, elle s'inscrit dans le prolongement de travaux d'auteurs comme Blanchard, Demangeon, ou Pierre George, elle inverse par ailleurs les termes de l'analyse classique des liens entre le fait urbain et la région, ou du moins elle en parachève le renversement. En effet, si certains géographes s'attachaient à définir des "familles de villes ", c'était sur la base d'une série de caractéristiques similaires héritées de l'histoire et du milieu naturel (ou de leurs relations), et en dernier ressort de l'inclusion dans un même espace : les villes d'une zone de piémont, de contacts, d'un littoral, d'un axe fluvial, d'un couloir de circulation, ou encore d'une "région » industrielle, agricole, etc. En d'autres termes, lorsque l'on tentait de qualifier les villes d'une "région ", on recherchait avant tout en quoi elles tiraient des éléments d'identité commune de leur appartenance à cette région, quelle que soit l'identité de celle-ci. Avec les premiers articles de Georges Chabot et de Pierre George, mais surtout avec la thèse de Michel Rochefort, la région cessera d'être conçue comme un cadre, en quelque sorte externe et préexistant aux villes situées à l'intérieur. C'est dans le fait urbain, et plus spécifiquement dans les relations des villes, - les unes avec les autres, avec leurs zones d'influence, et avec le monde extérieur - que l'on va appréhender l'identité, la consistance même de la région. 
Les villes ne sont plus définies en elles-mêmes, elles ne sont plus dissociées de l'organisation de l'économie régionale dans son ensemble, et la région trouve son unité fonctionnelle dans l'ensemble hiérarchisé des villes que constitue le réseau urbain ainsi que dans l'ensemble des relations et flux qui l'animent.

La région est saisie dans sa dynamique et sa diversité. Si son analyse permet de mettre en évidence les formes de concentration et de polarisation économiques dans l'espace et les disparités urbaines et régionales, la thèse donne aussi, on l'a trop souvent oublié, des clés pour comprendre les mécanismes socio-économiques d'organisation urbaine et régionale. Sur ce plan son approche est essentiellement historique, bien qu'elle débouche sur la période actuelle. Il s'agit d'appréhender les processus de formation des réseaux urbains et leurs configurations actuelles, et ceci nécessite une reconstitution minutieuse de l'évolution du développement des villes et de leur rôle. Mais l'analyse s'attache alors à saisir les villes à la fois dans leurs rapports à l'évolution globale de l'économie et dans leurs propres dynamiques internes. Elle pénètre dans le jeu des acteurs, et en particulier des bourgeoisies, mettant en évidence leur place dans le développement local et régional. Elle contribue simultanément à montrer concrètement comment la concentration du capital structure l'organisation urbaine, et la façon dont se manifeste, peu à peu, l'emprise des capitaux extérieurs sur l'économie régionale. Au delà de l'étude du secteur tertiaire et de la construction d'un "modèle ", - modèle qu'il développera dans le cadre des travaux sur les Métropoles d'équilibre, et diffusera un temps avec succès un peu partout dans le monde, et notamment au Brésil ${ }^{28}$ -, la thèse de Michel Rochefort trace donc les grandes lignes d'une approche dialectique des rapports société/espace à différentes échelles. Prendre en compte le poids des héritages, celui des structures et du système économique mais aussi la diversité des acteurs et l'articulation des processus à différentes échelles, tels sont quelques grands traits de sa démarche qu'il reprendra plus systématiquement par la suite.

Du rejet du fonctionnalisme à la recherche d'une géographie totale

Il est inutile de revenir en détail sur le rôle de Michel Rochefort dans l'évolution de la géographie et de la recherche urbaine depuis les années 70 . Ses travaux des dernières décennies sont beaucoup mieux connus d'un large public que ceux des années 50 et même des années 60 , et ils n'ont pas fait l'objet d'interprétations réductrices comme les précédents. Quant au rôle qu'il a joué et joue encore à travers ses fonctions dans l'université et divers organismes de recherche, nombre d'entretiens et de témoignages suffisent à en rendre compte. On se bornera ici à situer ses grandes orientations au regard des évolutions scientifiques des trente dernières années.

Le début des années 70 marque à n'en pas douter un tournant. C'est à la fois un élargissement et une remise en cause de certains aspects de ses recherches antérieures, et notamment de la façon dont il avait abordé la question des rapports entre le développement urbain et le développement régional, qui lui semble trop "fonctionnaliste ». Sa confrontation avec les réalités du Tiers-Monde et les débats de l'époque sur le développement auxquels il contribue activement n'y sont pas étrangers ${ }^{29}$. S'il ne développe sa critique du fonctionnalisme que plus tard, on retrouve par contre une réflexion, souvent rapide et claire, sur ce point dans de nombreux entretiens ou publications des années $1980-1990^{30}$. Que veut-il signifier par là ? On peut dire, schématiquement, qu'il évoque une attitude intellectuelle consistant à penser que les différentes unités spatiales qui constituent un ensemble territorial, entretiennent a 
priori, un ensemble cohérent d'interrelations. Or on sait que la géographie régionale classique faisait un usage extrêmement important des notions d'harmonie et d'équilibre, et qu'une des idées force des élèves de Pierre George, surtout lorsqu'ils ont acquis la culture dispensée par des historiens, comme Pierre Labrousse, Fernand Braudel, ou des philosophes comme Hypolite, est précisément que toute structure, toute combinatoire est simultanément organisée selon le principe de cohésion et d'équilibre, dans lequel chaque élément a sa fonction, et traversée par des tensions internes, des contradictions. Si, au terme d'une relecture des divers travaux de Michel Rochefort, on peut avancer que la prise en compte des dynamiques historiques, et donc des antagonismes sociaux et économiques, était déjà présente dans sa thèse, comme le montrent les chapitres souvent négligés sur la genèse de l'armature urbaine de l'Alsace, reste que la méthode d'analyse des structures spatiales proprement dites - localisation et identification des centres constituant "l'armature urbaine ", définition de leurs aires d'influence - est effectivement « fonctionnaliste ». Telle est en tout cas la réserve qu'il tient à énoncer après coup. Le fonctionnalisme, à ses yeux, réside donc dans le statisme excessif qui ressort de la méthode, en particulier dans l'insuffisante attention apportée aux conflits sociaux et à la capacité d'innovation technologique et économique inégale des détenteurs de capitaux et des élites, en bref aux disparités des dynamiques historiques, locales et régionales, même si la méthode permet de mettre en évidence certaines «concurrences». À cela il faut ajouter une précision complémentaire: lorsqu'il dénonce rétrospectivement le fonctionnalisme auquel son travail des années 50 et 60 a offert quelques cautions, il évoque en réalité la réduction à laquelle ont procédé, d'une part les décideurs - fonctionnaires et plus encore responsables politiques - en prenant appui sur les études d'armature urbaine pour répartir certaines aides de l'État à l'aménagement régional, d'autre part les chercheurs, qui ont mis l'accent de manière trop restrictive, au détriment d'autres dimensions de la réalité, sur l'analyse des données relatives au secteur tertiaire dans la recherche sur la logique des relations entre les villes et le développement. En fait, ce qui est fonctionnaliste, c'est moins une méthode d'analyse globale, qui attendait des prolongements, que l'application restrictive d'une partie de la méthode; la partie la plus formelle, la plus "spatialiste ", la moins ouverte à l'effort, nécessairement qualitatif et diachronique, d'interprétation des processus économiques, incluant leur articulation et leur dimension sociale et politique. C'est ce que signifie sans doute son expression elliptique de «fonctionnalisme du tertiaire ». Ceci étant, peut-on identifier et hiérarchiser les traits par lesquels Michel Rochefort se démarque de ce fonctionnalisme dans les articles et ouvrages qu'il a publiés depuis les années 70 ?

Ancrer la théorie dans la pratique

25 L'une des principales caractéristiques de sa démarche est l'alliance, sinon dans le court terme, du moins sur le long terme, entre empirisme et réflexion théorique, et en ce sens, on peut dire qu'il est resté fidèle à la ligne tracée par ses premiers "maîtres ", historiens des Annales ou géographes comme Pierre George et André Cholley.

Il n'a pas participé aux « révolutions » qui ont affecté la géographie anglo-saxonne et la géographie française à partir de la fin des années 60 et des années 70 . Tout en étant l'un des premiers en France à avoir consacré d'importantes parties de sa thèse à des analyses quantitatives, - qui ont fait école - il a toujours été prudent, même réservé, à l'égard du mouvement qui faisait de l'application de techniques statistiques de plus en plus élaborées le principal outil de la recherche, voire le principal moteur de l'innovation dans les études sur l'urbanisation et l'organisation de l'espace. Il n'a jamais 
manifesté d'hostilité envers le recours à la quantification et à la modélisation et envers les efforts visant à améliorer les méthodes mathématiques et cartographiques de l'analyse géographique. Au contraire, dans son esprit ces méthodes ont un intérêt heuristique et surtout elles contribuent à l'administration de la preuve et distinguent un véritable travail de recherche d'un simple essai : ceci est déjà très clair dans sa thèse. Mais il a toujours attaché une importance plus grande aux efforts plus complexes, et plus risqués aussi, que nécessite la reconstitution diachronique des processus et leur interprétation, impliquant de multiples va-et-vient entre observation empirique, réflexion abstraite, lectures, entretiens avec des acteurs divers, etc. Sans doute ses publications les plus notoires de la période de participation à la politique des métropoles d'équilibre n'ont-elles pas donné une image claire de ce souci de " comprendre les mécanismes ». Mais, sur l'ensemble de son activité, cette volonté a constitué une ligne directrice indubitable.

Cette posture intellectuelle, - conjuguant de fréquentes anticipations et une résistance systématique aux effets de mode, et donc à leur reflux, ainsi qu'un refus discret, mais ferme, de se comporter en chef de file -, apparaît plus nettement dans les rapports distanciés de Michel Rochefort avec les grands courants théoriques qui traversent l'économie, la sociologie ou la géographie dans les années 60 et 70. Inutile d'y revenir: il ne s'est pas posé en pur théoricien, même si, par leur concision et leur densité, plusieurs de ses ouvrages, - tels Le défi urbain dans les pays $d u S^{3} d^{31}$, ou ses articles comme sa contribution à Métropoles en déséquilibre - ont fait beaucoup plus que de nombreux ouvrages de spécialistes d'essais théoriques. Mais toutes ses publications, ou presque, témoignent du souci de ne jamais dissocier la théorie de la pratique et de cette préoccupation prioritaire : comprendre les mécanismes en jeu. Et l'une des raisons pour lesquelles ses écrits sont relativement peu nombreux et brefs au regard de leur influence scientifique réelle, est précisément l'idée qu'il existe une hiérarchie parmi les faits relevant du domaine de la géographie et des recherches sur l'aménagement et l'urbanisme. Cette hiérarchie relève de deux catégories de considérations, en réalité indissociables, et qui, toutes deux, ont trait au sens des faits : leur valeur explicative, d'un côté ; leurs incidences sociales, de l'autre.

La problématique, parfois elliptique, mais qui sous-tend toujours fortement ses écrits, repose donc à la fois sur une hiérarchie des problèmes d'interprétation et sur une hiérarchie des problèmes économiques et sociaux. Tout ceci est exprimé avec plus de précision dans un texte de 1984 que Michel Rochefort n'a pas rédigé personnellement, mais qu'il a co-signé, - l'avant-propos de l'ouvrage collectif Sens et non-sens de l'espace ${ }^{32}$ et ce n'est pas un hasard si certaines des idées essentielles de Michel Rochefort sur la recherche s'expriment dans ce texte collectif. On a là un fil directeur qui traverse tous ses travaux : sa thèse, mais aussi, malgré leurs aspects de " dérive fonctionnaliste », les études menées en collaboration avec Jean Hautreux et Lecourt; ses recherches et celles qu'il a impulsées sur le Tiers-Monde; enfin, ses travaux des années 90 sur l'aménagement et l'urbanisation. De toute évidence, à ses yeux, les progrès théoriques, qu'il s'agisse de recherche géographique ou urbaine, reposent sur l'étude de problèmes concrets, inscrits dans des contextes historiques et géographiques précis, ce qui ne signifie pas dans des cadres spatiaux nécessairement étroits, plus que sur des tentatives d'élaborer des constructions théoriques désincarnées sur des objets trop vastes pour ne pas donner lieu à un excès d'abstraction. Au fond, comme il le dit lui-même ${ }^{33}$, Michel Rochefort, tant pour des raisons affectives que théoriques, a toujours préféré le travail de terrain au travail de cabinet - c'est une des raisons pour lesquelles il continue à 
effectuer de nombreuses missions, au Brésil et en Tunisie notamment - même si les deux sont toujours complémentaires. Et, bien qu'il en ait fait usage, sa dénonciation du fonctionnalisme tient très certainement à sa défiance à l'égard de schémas ou de modèles trop simplificateurs, présentés comme "théoriques", et que l'on a trop souvent tenté d'appliquer comme s'ils avaient une portée universelle. En ce sens, c'est essentiellement à travers sa pratique de la recherche et de la direction de recherches, que l'on peut dégager les orientations théoriques de Michel Rochefort et leur évolution au cours des dernières décennies.

Aborder de front la complexité

Cette évolution ne constitue en fait nullement un revirement dans sa pensée, dans la mesure où dans sa thèse, et même dans ses travaux pour le ministère de l'Équipement, il n'avait pas mis de côté ses engagements initiaux. Mais on peut parler plus justement d'approfondissement. Son expérience personnelle au Brésil et la direction d'une équipe de chercheurs sur le Tiers-Monde, ont confirmé et affermi ses options politiques et intellectuelles des années 50, et ses travaux ultérieurs ont été pour lui l'occasion de les exprimer plus explicitement.

Une des constantes de ses orientations est l'attention qu'il porte aux incidences des mécanismes macro-économiques. Quels que soient la thématique et l'espace considéré, il importe avant tout de définir le plus rigoureusement possible en quoi et comment les phénomènes étudiés s'articulent au système productif global. Mais il ne s'agit pas de sacrifier à un déterminisme de l'économique sur le social ou sur le spatial, comme l'ont fait parfois certains chercheurs dont il était proche par ses questionnements et ses options politiques, ni à un déterminisme des emboîtements d'échelles - ce qui se passe à l'échelle locale dépendant de ce qui se passe à l'échelle régionale etc., jusqu'à l'échelle mondiale. Au contraire, il s'agit d'évaluer les marges de variations par rapport aux tendances globales, et plus particulièrement les spécificités locales, en portant l'analyse sur la dialectique des rapports à différents niveaux et différentes échelles. Et s'il voit une avancée théorique, une grille d'interprétation particulièrement féconde, dans l'idée selon laquelle les formes d'urbanisation et d'aménagement sont la "projection " dans l'espace des rapports sociaux, elle est pour lui une hypothèse. Celle-ci ne peut guider utilement le chercheur que dans la mesure où elle fait l'objet d'un travail de vérification empirique, où, sous la notion de rapports sociaux et celle de projection, est pris en considération tout un ensemble complexe de processus et où l'analyse ne procède pas de manière purement déductive. Ainsi, chercher à saisir dans quelle mesure le fonctionnement de l'économie globale intervient dans l'organisation locale ou régionale de l'espace, et comment chaque formation socio-spatiale y répond, est un des moyens d'échapper au risque du fonctionnalisme.

Décliner l'analyse à différentes échelles est par conséquent une des caractéristiques des orientations intellectuelles de Michel Rochefort, indissociable de son souci d'appréhender avec justesse le rôle des mécanismes macro-économiques dans l'organisation de l'espace et son évolution. Depuis 1970, dans pratiquement toutes les études de cas qu'il a menées personnellement, ou dont il a suivi de près la réalisation, on peut noter un effort pour tirer au clair les relations entre les structures et processus observables dans le cadre spatial considéré et les processus à l'œuvre à des échelles plus étendues, et en dernier ressort à l'échelle mondiale. Sans doute, l'élargissement des aires d'intervention des principaux acteurs économiques est-il alors déjà très avancé. Et il n'y a pas, dans la démarche de Michel Rochefort, d'innovation radicale sur 
des questions qui commencent à devenir classiques comme celles de l'impérialisme, de la domination des firmes multi-nationales, des liens entre le capitalisme international et la sphère du politique. Mais par son souci d'aborder ces articulations - l'un des concepts-clés de sa pensée - dans leur diversité et leur complexité, Michel Rochefort est un pionnier des problématiques qui se diffuseront massivement dans les années 80 et 90 . Ses analyses accordent en effet une place essentielle à la dialectique des rapports global/local - un des fils directeurs de sa réflexion -, même s'il ne s'attache pas à les théoriser et n'emploie pas ce vocabulaire. C'est précisément un des plans sur lesquels, en raison de son engagement dans la recherche sur le Tiers-Monde, l'approfondissement de sa démarche est le plus manifeste, par rapport à sa thèse. Dans celle-ci le monde extérieur n'occupait qu'une place limitée et l'analyse était centrée, de façon très restrictive, sur les structures économiques internes à la région. Autrement dit, n'étaient étudiés de manière approfondie que les rapports entre les échelons local, micro-régional, et régional, au détriment des rapports entre l'économie régionale et l'économie nationale - question qu'il n'avait fait qu'effleurer- et plus encore l'économie mondiale. Mais, si l'on se réfère à ses publications ultérieures, aurait-il publié, ne fût-ce que dix ans plus tard, un travail sur le même thème, sans consacrer d'importants développement à situer les enjeux économiques, locaux et régionaux, dans un rapport de force international ? Toujours est-il que, dès le début des années 70, dans tous ses travaux se manifeste le souci de définir les rapports de force à toutes les échelles, internes et externes.

Désormais les comparaisons et l'étude des rapports de force internes/externes au cadre géographique de l'espace étudié ont dans sa démarche une double fonction : ce sont des moyens d'approche sur le plan méthodologique, mais aussi des objets de recherche à part entière. D'un côté, on ne peut comprendre la spécificité des phénomènes observés dans un cadre spatial donné, sans saisir en quoi ces phénomènes relèvent de logiques de portée globale. Mais, d'un autre côté, on ne peut vraiment progresser dans la compréhension des lois de l'économie et de la société, sans s'appuyer sur la diversité des études de cas, locales, régionales, nationales. Elles apportent des compléments d'éclairages, une connaissance plus nuancée des grands mécanismes. Plus fondamentalement, les écarts, loin d'être de simples résidus des modèles, en sont des composantes essentielles. Un des ressorts de l'économie réside en effet dans les disparités d'un espace à l'autre. Dans la pensée de Michel Rochefort, telle qu'on peut la saisir à travers ses écrits et les témoignages des chercheurs qu'il a encadrés, les variantes géographiques, les disparités dans les formes et les niveaux de développement et d'organisation, sont autant d'éléments constitutifs de l'évolution économique. Et, de fait, ne constate-t-on pas que la globalisation de l'économie progresse en exploitant ces écarts? En effet, si le processus de globalisation suscite des tendances à l'uniformisation, (des structures économiques et sociales, de certaines pratiques culturelles, etc.), la diversité des espaces à toutes les échelles, est, plus que jamais, un vecteur du gonflement des échanges, une des raisons du rôle de plus en plus décisif du secteur tertiaire dans l'économie en général, qu'il avait pressenti dans ses premiers travaux, et peut-être aussi de l'imbrication des structures et des mutations politiques, économiques, sociales, imbrications que les travaux sur le Tiers-Monde ont si vivement mises en lumière.

33 Indissociable de l'étude des interactions entre le local et le global, l'identification des spécificités locales implique la prise en compte du poids de l'histoire, mais d'une histoire qui ne se limite pas au domaine de l'économie, même lorsque le thème central 
du questionnement est économique. Ses travaux sur le Brésil ont achevé de le convaincre que, si une nation, une région ou une ville réagit différemment des autres face au mouvement global de l'économie, c'est parce que son histoire lui a légué des structures - économiques, mais aussi sociales, politiques, culturelles, architecturales, environnementales même, etc. - particulières. La diversité des variantes dans l'espace renvoie donc directement à la diversité des temporalités : toute analyse géographique doit passer par une périodisation, par le repérage d'héritages et de ruptures. Mais on peut également noter que la façon dont Michel Rochefort conçoit les rapports entre les différentes échelles de l'analyse géographique est simultanément une conception qu'il a peu formalisée mais systématiquement mise en pratique - des rapports entre recherche à finalité théorique et recherche empirique, qu'il a toujours refusé de séparer. D'où l'importance qu'il accorde au jeu des acteurs dans un espace donné, dont la compréhension implique la prise en compte du poids de l'histoire économique, sociale, politique de l'espace en question, tout autant que l'histoire de sa position par rapport au monde extérieur. La part respective des contraintes dues au poids du système englobant et de l'autonomie du système local n'est pas le produit d'un déterminisme historique. Ce sont les rapports de force entre les acteurs et leurs stratégies qui suscitent les changements et les inflexions échappant à un tel déterminisme, mais ceux-ci s'inscrivent dans un processus historique. La démarche géographique, qui en cela s'écarte de celle de certains sociologues avec qui Michel Rochefort a beaucoup dialogué, inclut donc nécessairement un recours à l'histoire. Il est donc très fidèle à la géographie classique par ce recours à l'histoire, mais il se démarque d'une tradition qui réduisait la géographie à l'étude de la strate la plus récente de l'évolution historique. Son souci est de comprendre en quoi chaque formation socio-spatiale diffère ou ressemble à telle ou telle autre, et pour cela, l'analyse des processus, dont certains de longue durée est indispensable. Par là, on peut dire que ses travaux consécutifs à l'expérience brésilienne renouent, sans rupture, mais avec une netteté accrue par rapport à ceux sur les réseaux, avec sa double filiation intellectuelle : celle qui le relie à la philosophie marxisante, sinon marxiste, et celle qui le relie aux premiers historiens économistes de l'école des Annales. Sensibilité au poids de l'histoire, importance attachée aux variantes spatiales et aux stratégies des acteurs qui en assurent la dynamique : deux facettes, sinon d'une même défiance à l'égard des tentatives d'interprétation systémique, du moins de l'esprit de système. Peut-être y-a-til là une des raisons qui l'ont poussé à garder de la distance à l'égard d'un certain nombre de chercheurs d'inspiration marxiste. Il nous semble que cela a été le cas dans les années 70 , lorsque s'affirmait un "structuralo-marxisme", très attaché à la construction de modèles.

Le recours à une démarche plus historique, intégrant constamment dans l'étude des faits l'analyse des processus, des héritages et des ruptures, n'est qu'un des aspects d'une plus grande ouverture à la pluridisciplinarité, qui n'a fait que s'affirmer depuis cette période : approfondissement et non véritable inflexion, on l'a déjà relevé. Mais antérieurement, il s'agissait davantage d'une sensibilité que d'une véritable pratique pluridisciplinaire, car dans sa façon d'emprunter à l'histoire et à l'économie, il ne rompait pas avec les usages de la géographie, même si c'était à une conception moderne de l'histoire, celle des Annales, qu'il se référait, et même si les questions économiques qu'il abordait étaient relativement nouvelles. Dans les dernières décennies, ses contacts avec des chercheurs d'autres disciplines se sont en effet multipliés : non plus seulement avec des ingénieurs ou urbanistes appartenant aux 
milieux de la planification, mais avec des économistes, des chercheurs en urbanisme et des sociologues. Contacts et échanges de nature diverse : dans le cadre de publications collectives, mais aussi dans le cadre de commissions de réflexion, de programmation et d'animation de la recherche. Surtout, l'attention portée à la thématique et aux démarches des autres sciences sociales éclate, beaucoup plus que jadis, dans ses publications et dans ses directions de travaux. À tel point que l'on hésite sur la dénomination la plus adéquate pour parler du domaine sur lequel portent ses travaux: "géographie urbaine ", «études urbaines ", " aménagement et urbanisme »? Un des aspects les plus caractéristiques de cet élargissement est l'attention portée, non seulement aux processus, on l'a vu, mais plus précisément au jeu des acteurs, à leurs stratégies, à leurs rapports de force.

C'est au regard de cette inflexion que se pose la question des rapports entre Michel Rochefort et les sociologues du courant de sociologie urbaine marxiste française. Il n'y a pas lieu ici de présenter ce courant, qui s'est affirmé au début des années $70{ }^{34}$. Il suffit de rappeler que les travaux d'auteurs comme Manuel Castells, Jean Lojkine, ou encore Francis Godard, ont eu un retentissement considérable, bien au-delà du cercle des sociologues français, et ont inspiré de nombreux chercheurs ; en France et à l'étranger - notamment en Amérique Latine - en sociologie, mais aussi en géographie, en urbanisme et en économie. Qui plus est, leurs principales idées ont retenu l'attention de bon nombre de responsables d'études, voire de la planification, dans les milieux de la haute administration de l'aménagement du territoire et de l'urbanisme.

Leur faible influence sur la pensée et la pratique de Michel Rochefort justifie donc que l'on s'y arrête brièvement, d'autant que ces sociologues ne se contentent pas de se référer aux idées générales du marxisme, mais qu'ils affichent une volonté expresse de construire une œuvre théorique, actualisant et explicitant des éléments de la théorie marxiste que Marx lui-même n'avait pas développés, et qu'ils s'inspirent, directement ou indirectement, des travaux de Louis Althusser qui visaient à dégager des écrits de Marx une véritable philosophie. Autant de facteurs qui auraient pu susciter un plus grand intérêt de sa part. Le paradoxe est en fait plus apparent que réel, et il a expliqué dans cet ouvrage, pourquoi il cite rarement les articles et travaux des sociologues marxistes en question, et pourquoi leurs démarches l'ont, en fin de compte, peu influencé. En fait, d'une part, à l'époque où ce courant de pensée a émergé, il s'intéressait davantage aux problèmes d'aménagement dans les pays en voie de développement, surtout au Brésil, qu'aux questions urbaines françaises. De plus, cellesci étaient abordées essentiellement à l'échelle intra-urbaine, alors que lui s'intéressait surtout aux rapports entre l'urbanisation et l'ensemble de l'économie, à une échelle beaucoup plus étendue. Inversement, les premiers ouvrages, les plus « théorisants » du courant de sociologie marxiste, ne portent pas sur l'urbanisation dans le Tiers-Monde. Et, de ce fait, il était à certains égards en avance sur eux dans la construction de sa problématique, même si, encore une fois, il ne l'a pas toujours explicitement formulée.

En fin de compte, l'écart tient avant tout - comme il le dit lui-même - au fait que, même s'il estime indispensable de ne jamais couper le travail empirique de la réflexion théorique, même si, à ses yeux, toute étude de cas appelle un effort de généralisation, il se défie d'une pensée parfois quelque peu "mécanique ", excessivement déductive, insuffisamment attentive - pour reprendre un de ses leitmotivs - à la diversité et à la complexité du réel. Il faut enfin apporter une précision : il a toujours entretenu des relations intellectuelles importantes avec certains chercheurs qui, à bien des égards, tel 
Henri Coing, étaient affiliés au mouvement de la sociologie marxiste, mais sensibles aux variations des formes d'organisation et au poids des héritages historiques.

De même la thématique des travaux et des directions de recherche exprime-t-elle un élargissement, et non une véritable coupure. C'est toujours aux données économiques et à leur transcription spatiale - plus précisément à la façon dont l'économie imprime sa marque dans l'espace mais aussi dont l'espace modèle ou module l'évolution économique - que Michel Rochefort accorde la priorité. Mais, si l'on prend le seul exemple des relations entre les configurations spatiales des réseaux urbains et le développement économique, il suffit de parcourir ses livres et articles récents pour constater combien s'est étendue et enrichie sa problématique. En témoigne, entre autres exemples, la place occupée par un concept clé de son vocabulaire, celui de système productif. Dans des ouvrages comme Dynamique de l'espace français et aménagement du territoire ${ }^{35}$, l'emploi de la notion traduit clairement une conception de l'économie qui dépasse la segmentation entre les différents grands secteurs d'activité. Il s'agit au contraire de prendre en compte le fait que se multiplient les types d'entreprises dont la fonction économique et le mode d'organisation interne remettent en cause la distinction classique entre secondaire et tertiaire. Et, plus encore qu'une affaire de redéfinition de catégories classificatoires et statistiques dépassées, il s'agit de prendre en compte la complexification des mécanismes qui mettent les «fonctions productives » et les «fonctions de service » en étroite imbrication, mécanismes dont le gonflement du secteur «quaternaire » n'était qu'une des prémices. Enfin, la notion de système productif renvoie, simultanément, à la dimension sociale des mutations économiques, tant dans l'industrie que dans le commerce, la banque, ou les échanges : changements dans les modes d'organisation du travail, dans les hiérarchies de qualification, de statut et de rémunération, et, là encore, dans les relations, au sein même des entreprises, entre le travail ouvrier et les autres tâches. Si les premières publications de Michel Rochefort montraient bien son intuition de la montée des services, et pas seulement de la consommation, dans la société et l'organisation de l'espace, ses travaux récents placent le lecteur directement au cœur des imbrications de plus en plus étroites entre le domaine de la production et celui des services, ou encore entre le monde de la technologie et celui de la communication.

Faut-il souligner combien ses idées sur les armatures et les réseaux urbains se sont également enrichies. S'il est toujours question d'appréhender des hiérarchies entre les villes et les régions, ce n'est plus pour déterminer des configurations spatiales. Loin de tout « spatialisme ", l'objectif est de contribuer à la compréhension des phénomènes de domination. Sur ce plan encore, l'observation des processus, des changements, est désormais intégrée systématiquement à l'analyse. Et la géographie sociale est intégrée à la géographie économique: la grande question est de comprendre en quoi les disparités spatiales sont en interaction avec les inégalités sociales. Un signe parmi d'autres de l'évolution de sa pensée en ce domaine, au cours des deux dernières décennies, est que le terme de métropolisation apparaît plus fréquemment dans ses écrits que celui de métropole. Changement ténu, en apparence, mais recourir à la notion de " métropolisation » ou à celle d'espace métropolitain, plutôt qu'à des notions comme celles de métropole, de réseau, ou d'armature urbaine, autour desquelles étaient structurées les travaux des années 60 , est bien l'indice d'une attention plus systématique à la dynamique et au mouvement des formes d'organisation. À vrai dire ceci n'est pas totalement nouveau, et l'on sait qu'il a toujours préféré une géographie soucieuse de comprendre - et donc de saisir le mouvement - à une géographie 
soucieuse d'inventorier des faits et leurs formes de distribution, même si, un temps, il a consacré une partie importante de son travail à analyser certaines de ces formes.

En tout cas, la portée de cette inflexion dans le vocabulaire, apparaît clairement dans les débats du colloque « Métropoles en déséquilibre ». Comme le note Jean-Paul Lacaze, la notion même de métropole d'équilibre, telle qu'elle avait été définie dans les débuts de la Cinquième République - et l'on pourrait élargir ce jugement à l'essentiel des enquêtes sur l'armature urbaine - reposait sur une conception hiérarchique et statique des réseaux urbains. Sans doute était-il utile, et même nécessaire, dans un premier temps d'avoir conscience du poids des héritages historiques et des inerties dans la trame géographique des villes et de leurs relations ${ }^{36}$. Et pour Michel Rochefort, leur prise en compte, on l'a vu, est toujours essentielle. Mais à cette conception était également liée l'idée, pour ne pas dire le postulat fonctionnaliste, que les distorsions constatées et même mesurées entre la réalité existante et le "modèle idéal» de hiérarchie et de répartition des centres urbains, ainsi que de leurs aires respectives d'influence, était en partie responsable des dysfonctionnements de la croissance économique régionale. Et réciproquement on pensait qu'une politique d'équipement consistant à renforcer la domination des métropoles sur leurs régions, en rééquilibrant leur pouvoir économique, devait contribuer au développement des services et de l'industrie, et cela non seulement dans les agglomérations ainsi promues, mais dans l'ensemble des villes et régions situées dans leur rayon de commandement. Or, le postulat selon lequel la correction des imperfections de l'armature urbaine, et principalement de sa disposition spatiale, est un outil efficace de développement, relève très précisément de l'attitude fonctionnaliste, que critique Michel Rochefort. Et ce qui en justifie la critique, c'est une idée trop mécaniste, voire trop déterministe, du rôle de l'armature urbaine dans le développement. C'est une conception qui sousestime certaines mutations dont les données chiffrées masquent les incidences historiques réelles, et qui ne reconnaît pas suffisamment la capacité du capital, notamment dans le domaine industriel, à s'affranchir du relais local et régional. En caricaturant, on pourrait dire que c'est la conception qui a inspiré la construction des «centres directionnels» destinés à assurer l'emprise des «métropoles» sur l'industrialisation régionale dans les années 70 , à l'époque même où le capital, non seulement se dérégionalisait, mais commençait à s'internationaliser à grands pas. Le schéma christallérien érigé en modèle à l'ère des débuts de la globalisation... C'est ce constat de déphasage, entre une réalité mouvante et une représentation de l'espace demeurée formaliste et statique, qui inspire Michel Rochefort lorsqu'il souligne, selon Lacaze, la déconnexion entre « une organisation spatiale des sociétés qui repose sur des centres régionaux de services, surtout à l'usage des particuliers, et l'avènement d'un système nouveau, régi selon une organisation spatiale plus complexe, de relations entre les services aux entreprises et la décision ».

41 L'analyse des processus de métropolisation, plus attentive aux changements, aux innovations qu'aux structures héritées, vise donc, avant tout, à comprendre l'élargissement des échelles dans les échanges ainsi que dans les mécanismes d'investissements et de décision. Cette inflexion révèle en fait son intérêt pour les mutations qui se produisent, dans la seconde moitié du XXe siècle, dans les mécanismes de disparité géographique, à l'échelle des nations et des régions. Certes, il subsiste de multiples éléments de continuité entre la problématique de sa thèse et de ses travaux des années 90 ; la concurrence entre les villes et les disparités dans les formes et les rythmes de développement local ou régional demeurent des questions essentielles. 
Mais désormais l'accent est placé sur les flux, et notamment les "flux de décision », et la question des concurrences interurbaines se pose à une autre échelle. Dans Métropoles et fragmentation des espaces ${ }^{37}$, Michel Rochefort souligne par exemple que c'est "l'internationalisation des relations de commandement des firmes privées ", qui, de nos jours, constitue le facteur décisif en ce domaine. La globalisation « définit, et définira dans l'avenir, des pôles majeurs dans les espaces transnationaux ». Étudier la métropolisation, c'est donc étudier à la fois les mécanismes qui commandent les reclassements à l'œuvre dans la concurrence des principales agglomérations, et leurs conséquences sur les régions qui se structurent autour d'elles. Dès lors, la question est moins de continuer à chercher à comprendre en quoi l'évolution historique des relations entre les groupes sociaux a déterminé une hiérarchie interne entre les pôles urbains au sein d'une région, et les limites entre les sous-régions gravitant autour de ces divers pôles, mais bien davantage de saisir les processus qui déterminent l'émergence, le plafonnement ou le déclin des grandes métropoles, et les disparités économiques et sociales qui en résultent. Pour autant, l'attention aux processus de concurrence dont la logique globale relève de la "globalisation" n'exclut pas l'attention à leur dimension locale. À l'intérieur des grandes régions, les variations locales que l'on constate dans les structures et le rythme d'évolution du système productif ne sont pas qu'un résultat, important par ses incidences sociales, de l'inégale capacité des métropoles à se placer dans le jeu de la compétition "globale »; elles en sont également un élément actif, sinon déterminant. Et, bien évidemment, c'est à l'échelle «locale» ou «micro-régionale » qu'il convient de prendre en compte les implications sociales des mutations économiques, et notamment, d'analyser les relations entre les pouvoirs publics, les différentes catégories de firmes et les principaux groupes sociaux. L'étude de la métropolisation nécessite donc, une fois encore, une attention simultanée à l'articulation des échelles - ou plus exactement, à l'articulation des processus qui s'exercent à plusieurs échelles - à la jonction de l'économique, du social et du politique, mais aussi une attention systématique aux rapports entre les évolutions à long terme et les changements à plus court terme.

42 À cette orientation, qui témoigne de la fidélité de Michel Rochefort à ses préoccupations premières, sont liées d'autres inflexions. Il accorde depuis les années 70 une place essentielle aux réflexions et aux recherches sur les classes sociales, sur les inégalités et sur le rôle de l'État dans l'organisation de l'espace.

43 La présence de l'État était déjà sensible dans ses premiers travaux. La question était abordée sous différents angles, des structures territoriales du pouvoir, du tracé des frontières aux tensions entre traditions centralisatrices et tentatives de décentralisation, jusqu'au choix des «métropoles d'équilibre "... Ce n'est toutefois qu'à partir de son engagement dans la recherche sur le Tiers-monde que les questions du pouvoir politique et de son rôle prennent toute leur place dans sa problématique. Concernant les pays en développement, il insiste sur l'importance des interactions entre les relations politiques et les relations économiques internationales (échanges commerciaux, flux de capitaux, etc.), et rappelle à quel point il est indispensable de prendre en compte les données politiques lorsque l'on cherche à identifier la logique des disparités du développement à l'échelle des nations. Non moins remarquable est l'attention plus systématique qu'il attache à la dimension politique des faits, sur les questions relatives aux villes et à l'organisation de l'espace, que ce soit en France ou dans les pays en développement. Le souci constant, évoqué plus haut, de resituer toute analyse à l'échelle locale, régionale ou nationale, dans un contexte plus étendu, et, en 
dernier ressort, de la rapporter aux processus de globalisation, conduit nécessairement à prendre en considération les comportements du pouvoir: même si les tendances économiques "lourdes " l'emportent à long terme, leurs modalités varient à court terme, selon les réglementations et les décisions des instances politiques. En résumé, pour Michel Rochefort la compréhension des écarts entre un "modèle " général d'évolution et ses variantes géographiques est partie intégrante de la construction d'un modèle. Une des conséquences de cette option méthodologique est que l'on resterait à la surface des choses si l'on se contentait de décrire les variations spatiales des indicateurs économiques et sociaux sans analyser la logique des acteurs et de leurs interactions.

L'État, le pouvoir local, sont donc parmi les acteurs les plus importants, et une recherche qui se cantonnerait dans l'étude de l'action des entreprises, sans chercher à saisir leurs relations - de solidarité et de connivence, ou d'antagonismes éventuels avec les pouvoirs politiques ne saisirait qu'une pellicule de la réalité. Mais à l'opposé, Michel Rochefort se défie des travaux trop restrictivement axés sur l'étude des décisions politiques, en matière d'action économique, d'aménagement régional, ou d'urbanisme. L'étude des décisions, même diachronique, ne saurait passer pour une étude des "politiques " : celle-ci, pour être complète, doit inclure l'étude de l'action concrète engagée, des obstacles qu'elle rencontre, des écarts entre les projets et les réalisations, des stratégies contradictoires des acteurs etc. Il est certes fondamental de saisir les bases et les implications politiques et juridiques de l'aménagement, mais se cantonner dans l'analyse des textes législatifs et réglementaires et dans celle des structures institutionnelles du pouvoir, y compris du pouvoir local, ou du moins en surestimer l'influence sur la réalité urbaine et territoriale, comme on peut l'observer parfois dans l'enseignement et la recherche, c'est courir le risque d'une dérive vers le fonctionnalisme.

Enfin, dans le même esprit critique, une dernière caractéristique tout aussi fondamentale de l'œuvre écrite et orale de Michel Rochefort, réside dans sa façon de mettre l'accent sur la dimension proprement sociale de tout ce qui touche à l'urbanisme et à l'espace. On se souvient du soutien qu'il a apporté en 1984, à la tentative menée par un certain nombre de géographes de susciter un mouvement en faveur d'une nouvelle " géographie sociale ». Il n'y a pas lieu ici de discuter du réel degré d'innovation, ni du renouvellement méthodologique effectivement apporté par les participants à ce mouvement. On retiendra du moins que, dans sa participation aux débats qui ont précédé et suivi l'ouvrage collectif Sens et non sens de l'espace $e^{38}$, dont le sous-titre, De la géographie sociale à la géographie urbaine, justifierait un long commentaire, Michel Rochefort a appuyé l'idée selon laquelle l'étude des formes d'organisation de l'espace, à quelque échelle que ce soit, n'a de sens que si elle fait une large place à la diversité des structures, des besoins et aspirations, des formes d'expression et moyens d'action politique et économiques des groupes sociaux, et notamment des classes sociales. Et que ce soit dans ses articles et ses ouvrages, son enseignement, ses contributions orales, ou ses fonctions d'animateur de la recherche, il a manifesté une attention toujours plus précise, au rôle des différentes catégories d'acteurs, et pas seulement des acteurs collectifs, politiques, économiques, mais aussi des acteurs que seule une observation micro-sociologique et micro-géographique peut comprendre. Cette préoccupation apparaît aussi bien lorsqu'il traite des inégalités et de 
l'exclusion, que lorsqu'il prône des analyses sur les leaders d'opinion et les individus qui lancent de nouvelles pratiques ou contribuent à leur diffusion.

Depuis longtemps très attentif aux phénomènes de domination et d'oppression dans le Tiers-Monde, il n'a cessé d'en approfondir l'étude. Mais loin de se contenter de les décrire et de les dénoncer, il marque son souci d'analyser la marge de liberté dont les groupes dominés, et parmi eux les plus pauvres, peuvent disposer pour développer des stratégies autonomes. S'il met en évidence les contraintes qu'impliquent la globalisation de l'économie et la réorganisation de ses liens avec le pouvoir, à toutes les échelles, il cherche aussi à montrer en quoi et comment de nouvelles formes de résistance, de lutte, et d'innovation dans les pratiques, participent à la dynamique du changement socio-spatial. Sans jamais céder à l'illusion, son œuvre est traversée par l'idée que les rapports sociaux et les formes territoriales, loin d'enfermer les individus et les groupes dans des systèmes de contrainte totalement clos, laissent ouvert le champ des possibles. On retrouve là tout le sens et la portée de la pensée et de la démarche de Michel Rochefort. Mettre en jeu les rapports de force contradictoires qui animent le mouvement des sociétés et des espaces, en plaçant les rapports sociaux et les stratégies des acteurs au centre de l'analyse; aller toujours plus loin dans la complexité et la diversité des situations sans perdre de vue les tendances lourdes et les pesanteurs qui commandent les évolutions ; évaluer les marges d'action possibles et les évolutions porteuses de progrès. Tout ceci apparaît très clairement dans la conclusion de son livre le plus récent, Le défi urbain dans les pays du Sud ${ }^{39}$ :

Ceux-ci (les pays du Sud) peuvent-ils trouver dans les miettes laissées par les économies des pays développés en cours de mondialisation, les ressources nécessaires pour intervenir dans le cadre d'un développement durable, adapté aux problèmes spécifiques de leurs villes ? [...] De toutes façons la solution, si elle existe, ne viendra pas des influences extérieures, [...] Au lieu de considérer la pauvreté comme le mal qu'il faut préalablement combattre avant toute amélioration des villes, ce qu'on ne sait pas faire, il faut essayer de comprendre que d'autres manières de 'vivre la ville' peuvent s'organiser dans le cadre de la pauvreté, dans la mesure où celle-ci n'est pas une situation de misère totale et où elle laisse aux habitants la possibilité de réinventer leur cadre urbain. Cette marche possible vers un avenir meilleur se heurte pourtant à un problème non résolu, celui des différences de temporalité entre les actions et les besoins. Gouvernances locales ou pouvoirs centraux agissent dans des temporalités courtes alors que les programmes d'amélioration des espaces urbains demandent des temporalités longues. [...] Dans l'évolution même des sociétés, la participation, la solidarité, supposent aussi une temporalité longue pour engendrer un progrès de la citoyenneté. Or c'est à court terme qu'il faudrait apporter une réponse aux violences urbaines et en particulier au désespoir d'une jeunesse sans horizons. l'urbanisation du monde et de la réorganisation des espaces. 


\section{NOTES}

1.Voir, par exemple, le livre récent paru sous la direction de Thierry Paquot, Michel Lussault et Sophie Body-Gendrot, La ville et l'urbain, l'état des savoirs, La Découverte, Paris, 2000.

2.Si le marxisme avait déjà profondément imprégné l'histoire, et dans une bien moindre mesure la géographie, son influence s'y manifestait encore de façon diffuse. Cf. J. Le Goff, R. Chartier, J. Revel (dir.), La nouvelle histoire, Les encyclopédies du savoir moderne, Paris, 1978 ; Marxisme et géographie urbaine, Villes en parallèles, Université de Paris X, n 7, déc. 1983 ; Autour de Raymond Guglielmo, Géographie et Contestations, Université de Paris VIII, mars 1990.

3.Les Annales d'histoire économique et sociale, revue créée par Lucien Febvre et Marc Bloch en 1929, en rupture avec l'histoire évenementielle et politique et rebaptisée en 1946 Les Annales, Économies, Sociétés, Civilisations.

4.Cf. Michel Rochefort, La plaine d'Autun et ses bordures, étude de géographie agraire, DES sous la direction de Jean Tricart, Strasbourg, 1949, et « La pénétration des capitaux bourgeois dans la campagne autunoise, ses conséquences sur l'habitat et la structure agraire ", Revue de géographie de Lyon, 1950, 25, réédité dans cet ouvrage, ainsi que l'analyse qui en est faite précédemment par Françoise Plet dans son article, « Michel Rochefort et la Bourgogne».

5.Pierre George, qui a pourtant dirigé son travail, dira par la suite, que « sa méthode procède plus de l'enregistrement d'effets que de celui de processus moteurs ». 6.Terme employé par Michel Rochefort. Lui-même, ainsi que Guy Burgel dans son essai sur marxisme et géographie urbaine, en ont déjà donné quelques éléments d'explication. Pour autant on ne saurait minorer d'autres aspects de sa thèse. 7.La première Chaire de géographie à la Sorbonne date de 1890 (Vidal de la Blache), mais ce n'est qu'en 1940 qu'est créée une licence de géographie « partiellement détachée » de l'histoire, puis en 1944 une agrégation de géographie. Cf. Claude Bataillon, Pour la géographie, Flammarion, Paris, 1999.

8.Les historiens des Annales avaient une conception de l'histoire, qui en faisait d'ardents défenseurs de l'interdisciplinarité, ce qu'illustre la composition du Comité scientifique de la revue à ses débuts, où l'on compte un géographe, A. Demangeon ; un sociologue, M. Halbwachs ; un économiste, C. Rist ; un politologue, A. Siegfried et quatre historiens (Cf. J. Revel et R. Chartier, La nouvelle histoire, op. cit., et J. Revel, « Histoire et sciences sociales, une confrontation instable » Passés composés, champs et chantiers de l'histoire, Autrement, série Mutations, $\mathrm{n}^{\circ} 150-151$, janvier 1995).

9.Voir notamment les travaux d'André Cholley, directeur de l'Institut de géographie de Paris de 1944 à 1956, un des géographes qui ont marqué Michel Rochefort à ses débuts. Cf. Le guide de l'étudiant en géographie, PUF, Paris, 1942, et « Problèmes de structure agraire et d'économie rurale », Annales de géographie, avril-juin 1946.

10.Guy Burgel, entretien avec Michel Rochefort, 1983, op. cit.

11.Cf. les travaux de Jean Dresch et Pierre George, et notamment La ville : le fait urbain à travers le monde, Paris, 1952, et l'article de Jean-François Deneux, « La ville : une relecture », in Marxisme et géographie urbaine, Villes en parallèle, op. cit.

12.Cf. à ce propos l'entretien de Michel Rochefort avec Guy Burgel, in G. Burgel, Marxisme et géographie, op. cit. 
13.Ceci l'avait conduit à préciser la notion de "région économique » et à poser la question des concurrences inter-urbaines et celle des limites d'extension du rôle d'une ville. Cf. Raoul Blanchard, (1877-1965), Grenoble, Étude de géographie urbaine, Armand Colin, Paris, 1911.

14.Georges Chabot, (1890-1975), «Les zones d'influence d'une ville », Communication au Congrès de Paris, session consacrée aux agglomérations urbaines, 1931. Dans ce texte il souligne : « On ne peut aujourd'hui se contenter d'opposer la ville [...] et la campagne. [...] La ville est devenue en effet le principe de groupement régional : le pays tout entier n'est que le champ des rivalités de ville à ville, et il est souvent utile de connaître jusqu'où s'étend l'influence d'une ville [...]. Mais il arrive plus fréquemment encore que l'influence d'une ville s'exerce par l'intermédiaire d'une autre [...]. Toute une hiérarchie s'établit ainsi de villes vassales et de villes suzeraines, celles-ci dépendant elles-mêmes les unes des autres. »Cf. la réédition de ce texte in $\mathrm{Ph}$. Pinchemel, M. C. Robic, J. L. Tissier, Deux siècles de géographie française, Comité des travaux historiques et scientifiques, Paris, 1984.

15.Georges Chabot, Les villes, Armand Colin, Paris, 1948 et « L'armature urbaine en géographie régionale », Urbanisme et Architecture, études en l'honneur de P. Lavedan, 1954.

16.Jean Gottmann, « Méthode d'analyse de Géographie humaine », Annales de géographie, LVI, n³01, janvier 1947 ; «L'aménagement de l'espace, Planification régionale et géographie ", Cahiers FNSP, 1952 ; Essais sur l'aménagement de l'espace habité, Mouton, Paris, 1966.

17.Jean Dresch, «Les villes congolaises. Étude de géographie urbaine et sociale », Revue de géographie et d'ethnologie, juin 1947 ; «Les villes d'Afrique occidentale », Cahiers d'Outre-mer, nº 11, 1950 ; « Recherches sur les investissements dans l'Union française d'Outre-mer ; leur répartition, leurs conséquences », Bulletin AGF, p. 231-232, 1953. 18.D'après un recensement de Numa Broc, sur 47 thèses soutenues entre 1917 et 1941, quatre seulement sont explicitement consacrées à l'étude de grandes villes, quelquesunes à de grandes régions urbano-industrielles. Cf. Numa Broc, « Homo geographicus : Radioscopie des géographes français de l'entre-deux-guerres » (1918-1939), Annales de Géographie, Paris, mai-juin 1993.

19.Cf. Rapports et travaux sur la décongestion des centres industriels, Délégation Générale de l'Équipement National, 1943-1945. Ces recherches associaient sociologues, économistes et géographes. On en retrouve l'essentiel dans le livre de Gabriel Dessus, Pierre George, Jacques Weulersse, Matériaux pour une géographie volontaire de l'industrie française, Cahiers de la Fondation Nationale de Sciences politiques, Armand Colin, Paris, 1949 ; voir également, Pierre George, Le métier de géographe, un demi-siècle de géographie, A. Colin, Paris, 1990.

20.Michel Rochefort, L'organisation urbaine de l'Alsace, Les Belles Lettres, Paris, 1960.

21.On se reportera au compte-rendu qu'en a fait Pierre George, qui est réédité dans cet ouvrage, et aux diverses contributions sur les suites apportées à ce travail, et notamment les recherches menées dans le cadre du ministère de l'Équipement sur les métropoles d'équilibre.

22.Même si, une dizaine d'années plus tard, Etienne Juillard qualifiera cette thèse de " révolutionnaire » en géographie, car elle permettait de comprendre à travers « ces flux invisibles que constitue la circulation des capitaux, [...] les ressorts de la mise en valeur d'une région ». Cf. E. Juillard, La "région ", contributions à une géographie générale des espaces régionaux, Ophrys, Paris, 1974. 
23. Michel Rochefort, L'organisation urbaine de l'Alsace, op. cit.

24.François Perroux qui a développé la notion de pôles de croissance, a été le chef de file du développement de l'économie régionale et appliquée dans les années 50 et 60 . Ses travaux et ceux de ses disciples, ont beaucoup influencé l'aménagement en France et dans le Tiers-Monde. Cf. François Perroux, "La notion de pôle de croissance ", Économie appliquée, $\mathrm{n}^{\circ} 1 / 2,1955$; «La firme motrice dans une région, et la région motrice ", Cahiers de l'ISEA, n 1, 1961 ; L'Économie du XX ${ }^{e}$ siècle, PUF, Paris, 1961. Jacques Boudeville avait appliqué la théorie des pôles de croissance aux cas brésilien et français, et entamé dans les années 60, avec Michel Rochefort, un rapprochement entre les géographes et les économistes. Cf. le recueil de textes, associant Michel Rochefort et Jean Labasse aux économistes, qui donne un bon aperçu de ces travaux et des débats entre géographes et économistes dans les années 60, Jacques Boudeville (dir.), L'espace et les pôles de croissance, Bibliothèque d'économie contemporaine, PUF, Paris, 1968. 25.Serge Antoine et Gérard Weill, chargés de missions à la DATAR, in Jacques Boudeville, L'espace et les pôles de croissance, op. cit.

26.Il souligne dans sa conclusion : " Pour interpréter toute ville, il ne suffit plus de la replacer dans son réseau régional ; il faut saisir les liens de structure qui subordonnent celui-ci à une unité plus vaste constituée par l'État ou par la zone d'influence de quelques grands trusts. La véritable unité de structure pour une géographie comparée des villes tend à dépasser le cadre du réseau urbain régional. » $C f$. Michel Rochefort, L'organisation urbaine de l'Alsace, op. cit.

27.On sait que Vidal de la Blache avait pressenti le rôle des grandes villes comme facteur d'unité régionale et que, depuis, l'idée avait été reprise par ses disciples, Blanchard dans son travail pionnier sur Grenoble, et l'historien Hauser, et surtout par Chabot, sans parler des travaux de Walter Christaller, dont la notoriété ne prendra toute son ampleur en France qu'après sa traduction en anglais, et après la publication des premiers travaux de la « nouvelle géographie », c'est-à-dire quelques années après la publication de la thèse de Michel Rochefort.

28.Le fait que cet aspect de son travail ait rencontré un tel écho au Brésil dans les années qui suivent la publication de sa thèse ne doit pas surprendre. C'est une époque où la géographie urbaine est encore relativement peu développée au Brésil et où certains géographes reconnus, français notamment, prônent encore les monographies urbaines et régionales. Et parallèlement, c'est un moment où les préoccupations en matière de développement et d'aménagement commencent à attirer l'attention sur les travaux d'économie régionale.

29.Voir par exemple sur cette question, André Gunder Frank, « Fonctionnalisme et dialectique ", Le développement du sous développement, l'Amérique Latine, Maspero, Paris, 1970.

30.On peut en lire des formulations particulièrement éclairantes dans ses contributions, souvent très denses, à des ouvrages collectifs, comme « Des Métropoles d'équilibre aux métropoles d'aujourd'hui », Métropoles en déséquilibre ? Paris, Economica, 1993, (reproduit dans cet ouvrage), ou encore « Métropoles et fragmentation des espaces " premier article du livre de textes réunis par Jean-Claude Driant, Habitat et villes : l'avenir en jeu, L'Harmattan, Coll. Villes et entreprise, Paris 1992.

31. Michel Rochefort, Le défi urbain dans les pays du Sud, Paris, L'Harmattan, 2000.

32.Sens et non sens de l'espace. De la géographie sociale à la géographie urbaine, Université de Paris X, 1984.

33.Voir dans cet ouvrage l'entretien avec Jeanine Cohen. 
34.Cf. Michel Amiot, Contre l'État, les sociologues, Paris, EHESS, 1986 et B. Lepetit et C. Topalov (dir.), La ville des sciences sociales, Paris, Belin, 2001.

35.Michel Rochefort, Dynamique de l'espace français et aménagement du territoire, Paris, L'Harmattan, 1995.

36. Ces inerties ont été bien analysées dans plusieurs ouvrages et articles de Denise Pumain, Thérèse Saint-Julien et, plus récemment, de France Guérin.

37.Michel Rochefort, Métropoles et fragmentation des espaces, op. cit.

38.Sens et non sens de l'espace. De la géographie sociale à la géographie urbaine, op. cit.

39.Michel Rochefort, Le défi urbain dans les pays du Sud, op. cit.

\section{AUTEURS}

JACQUES BRUN

professeur à l'université de Paris 1 , Ladyss

\section{CATHERINE PAIX}

Ladyss, Cnrs 